\title{
Development of an Effective Routing Protocol for Cluster Based Wireless Sensor Network for Soil Moisture Deficit Monitoring
}

\author{
Kamlendu Kumar Pandey and Dr. S.V. Patel \\ MSc IT Programme, Dept of Computer Science \\ Veer Narmad South Gujarat University, Surat \\ kamlendup@gmail.com,patelsv@gmail.com
}

\begin{abstract}
The use of wireless sensor networks in agriculture is anticipated to revolutionalise its operational mechanism. Many activities like moisture deficit monitoring, crop yield estimation and soil erosion can be automated using sensors. However, we need to develop deployment strategies, routing schemes appropriate for such applications. This paper focuses on special case of irrigated agriculture in gravity and pressurised irrigation. We have formulated a deployment strategy and developed an efficient routing protocol LATHAR (Location aware Threshold Based Hierarchical and Adaptive Routing) for transmitting the data packets from sensor node to the access point. The protocol has been tested on simulation model developed in $n s 2$ simulator and it has been found to perform best in almost all the matrices used to evaluate the performance of WSN.
\end{abstract}

Keywords: We Wireless Sensor Networks, Routing Protocol, irrigation

\section{Introduction}

The wireless sensor networks is one the products which are seen as the next-gen alternative to solve problems of redundant nature. With the advancements and maturity of this technology, the world is looking towards it to solve many existing problems like environmental monitoring, vehicle tracking, defense surveillance, early warning system for natural disasters, flood control, irrigation etc. It has got a very promising use in controlled irrigation systems. The efficiency of any controlled irrigation system depends on the actual demand of water and supply to replenish the demand, there has been always a gap between them due to inaccuracy in predicting the exact demand. The soil moisture sensor when interfaced with sensor mote was deployed as sensor node to sense the soil moisture. Deciding sensor topology and routing the sensed data was a major issue. After careful study of all the protocols it was decided to go for a new protocol. The new protocol was developed in a simulated environment and was tested for the crucial parameters with all other protocols. This paper is organized as

The rest of the paper is organised as follows

2) Related Work

3) WSN Topology and deployment of sensors

4) LATHAR - New Protocol development

5) Simulation

6) Result

7)Conclusion

\section{Related Work}

Many routing protocols have been used and conceptualized in field of wireless sensor networks[1]. These routing protocols are mainly classified based on Data Centric approach, Hierarchical Approach, Location based approach, Network and QoS flow based 
approach. The data centric approach focuses on gathering the data from randomly deployed huge no of sensors and finally getting the data at the sink. The approach of flooding [2] and gossiping does not need any routing protocol. In flooding, each sensor receiving a data packet broadcasts it to all of its neighbors and this process continues until the packet arrives at the destination or the maximum number of hops for the packet is reached. On the other hand, gossiping is a slightly enhanced version of flooding where the receiving node sends the packet to a randomly selected neighbor, which picks another random neighbor to forward the packet to and so on. Although flooding is very easy to implement, it has several drawbacks like implosion caused by duplicated messages sent to same node, overlap when two nodes sensing the same region send similar packets to the same neighbor and resource blindness by consuming large amount of energy without consideration for the energy constraints. Gossiping avoids the problem of implosion by just selecting a random node to send the packet rather than broadcasting. However, this cause delays in propagation of data through the nodes. The energy aware SPIN [3] (Sensor Protocol for information via negotiation) is based on the meta data attribute which is given to every node. The node negotiates with the neighboring nodes for Advertisement, negotiation and Data. The interested nodes will create a gradient toward Sink. The Directed Diffusion[4], an improvement to SPIN, was event and query based protocol where each node was told to store the data for a particular event. The query sending process established gradients from each node to the sink. When node gets the required data it will send to the sink from one of the several path selected dynamically. This saves more energy than SPIN. Destination-Sequenced Distance-Vector Routing (DSDV) [5] a table-driven routing scheme for ad hoc mobile networks based on the Bellman-Ford algorithm was developed by C. Perkins and P. Bhagwat. The main contribution of the algorithm was to solve the routing loop problem. Each entry in the routing table contains a sequence number, the sequence numbers are generally even if a link is present; else, an odd number is used. The number is generated by the destination, and the emitter needs to send out the next update with this number. Routing information is distributed between nodes by sending full dumps infrequently and smaller incremental updates more frequently. In AODV[6], the network is silent until a connection is needed. At that point the network node that needs a connection broadcasts a request for connection. Other AODV nodes forward this message, and record the node that they heard it from, creating an explosion of temporary routes back to the needy node. When a node receives such a message and already has a route to the desired node, it sends a message backwards through a temporary route to the requesting node. The needy node then begins using the route that has the least number of hops through other nodes. Unused entries in the routing tables are recycled after a time. The TORA [7] attempts to achieve a high degree of scalability using a "flat", non-hierarchical routing algorithm. In its operation the algorithm attempts to suppress, to the greatest extent possible, the generation of far-reaching control message propagation. In order to achieve this, the TORA does not use a shortest path solution, an approach which is unusual for routing algorithms of this type. TORA builds and maintains a Directed Acyclic Graph (DAG) rooted at a destination. No two nodes may have the same height. Information may flow from nodes with higher heights to nodes with lower heights. Information can therefore be thought of as a fluid that may only flow downhill. By maintaining a set of totally ordered heights at all times, TORA achieves loop-free multipath routing, as information cannot 'flow uphill' and so cross back on itself. Dynamic source routing protocol (DSR) [8] is an on-demand protocol designed to restrict the bandwidth consumed by control packets in ad hoc wireless networks by eliminating the periodic table-update messages required in the table-driven approach. The major difference between this and the other on-demand routing protocols is that it is beacon-less and hence does not require periodic hello packet (beacon) transmissions, which are used by a node to inform its neighbors of its presence. The basic approach of this protocol (and all other on-demand routing protocols) during 
the route construction phase is to establish a route by flooding RouteRequest packets in the network. The destination node, on receiving a RouteRequest packet, responds by sending a RouteReply packet back to the source, which carries the route traversed by the RouteRequest packet received. One of the important protocols in this was LEACH (Low Energy adaptive clustering Hierarchy ) [9]. It is a hierarchical protocol where $20 \%$ of the nodes are the candidate for the cluster head and looking to the energy level of the nodes one of them was selected as cluster he ad. The cluster head changed dynamically over a period of time thus not depleting the energy of the current cluster head. PEGASIS [10] was a improvement over LEACH. Rather than forming multiple clusters, PEGASIS forms chains from sensor nodes so that each node transmits and receives from a neighbor and only one node is selected from that chain to transmit to the base station (sink). Gathered data moves from node to node, aggregated and eventually sent to the base station. The next modification were TEEN [11] and APTEEN [12] protocols. Threshold sensitive Energy Efficient sensor Network protocol (TEEN) is a hierarchical protocol designed to be responsive to sudden changes in the sensed attributes such as temperature. Responsiveness is important for time-critical application. The Adaptive Threshold sensitive Energy Efficient sensor Network protocol (APTEEN) is an extension to TEEN and aims at both capturing periodic data collections and reacting to time critical events.

The protocols mentioned above do not take care any spatial parameters like postion or distance. The sole criteria of deciding the cluster head is energy. There are two reasons to go for a new protocol

1)The problem if moisture monitoring requires spatial distribution of the parameter

2) The area in consideration is large and sensors are located quite sparse as compared to the above protocols. As the relay limit of radio antenna in sensor networks is quite limited the distance between the node become a important factor for deciding the cluster head. The cluster head must me located at the optimal distance where all the sensing nodes can access them. The energy consideration on nodes is also combined to decide the cluster head.

So this paper presents a protocol suitable for wireless sensor network especially deployed to monitor moisture deficit in the irrigated area of gravity and pressurised irrigation

\section{WSN Topology and Deployment of Sensors}

The topology plays a very important role in placement of sensors and decide the correct routing or aggregation strategy. In case of irrigation random placement of sensor is not desired as the area is larger and moisture status as the sensed parameter does not vary rapidly in time but take a considerable duration. It varies from crop to crop and season to season. Also looking at the cost and the placement effort required to place sensors, predefined locations must be decided.

In case of canal irrigation, the subchak is the basic unit to calculate the irrigation demand while in pressurized irrigation the spacing of lateral and manifolds decide the layout and placement of sensors. In case of subchaks in canal irrigation the sensors are placed at a predefined locations to cover the entire area

While in Pressurized irrigation (drip irrigation) three sensors are placed in the line of laterals, one at the head second in the center and third one in the tail. One line of sensors represent two lines of lateral as shown in fig

The calculations to decide the no of sensors in drip irrigation is as follows :

No. of sensors in a drip irrigation layout $=[\{(\mathrm{Lm} / \mathrm{Sl})+1\}]^{*} 0.5^{*} \mathrm{~ns}$ for one side Lateral

No of sensors in a drip irrigation layout $=[\{(\mathrm{Lm} / \mathrm{Sl})+1\}] *$ ns for both side Lateral

Where Lm = Length of Manifold

$\mathrm{Sl}=$ Spacing of Lateral

$\mathrm{ns}=$ no of sensors placed on one lateral 
If the manifold head is taken as reference coordinate $(0,0)$. The sensors location can be determined by the lateral spacing and length as under

$\mathrm{Sn}(\mathrm{x}, \mathrm{y})=\mathrm{x}(0) \pm \mathrm{L} / 3, \mathrm{x}(0) \pm 2 \mathrm{~L} / 3, \mathrm{x}(0) \pm \mathrm{L}$

$\operatorname{Sn}(\mathrm{x}, \mathrm{y})=\mathrm{y}(0)+\mathrm{n} * \mathrm{Sl}$

$\mathrm{n}=\mathrm{Lm} / \mathrm{nL}$

Where $\mathrm{Sn}=$ sensor Node

$\mathrm{L}=$ Length of Lateral

$\mathrm{nL}=\quad$ No of Lateral on one side of Manifold

The cluster area in this case consists of block of 10 manifold outlets which comes to be approximately 30 sensors

For purpose of simulation Subchak is taken as one cluster block and in case of drip irrigation a block of 10 lateral is must be taken as a cluster block.

\section{LATHAR - (Location Aware Threshold Based Hierarchical and Adaptive Routing) - The New Routing Protocol}

Looking to all the popular protocols like AODV, DSR, DSDV, TORA, LEACH as given above in the related work, they seem to have been developed for the random sensor placements and the sensed data is of continuous nature. While AODV, DSR, DSDV and TORA have a non clustered LEACH provide the solutions for the cluster based sensors but taking $20 \%$ of sensors on adhoc basic does not satisfy the nature of the problem in considerations. As the distances are comparatively larger among the sensors the distance should also be a deciding factor. The dissemination interval is the another important factor. As the moisture data varies slowly keeping low dissemination does not give any change in the result. So the scheme of event based threshold model is combined with LATHAR to decrease the no of packet transmission and thus conserve the energy. LATHAR follows the following algorithm

\subsection{Algorithm:}

1. Get the dimensional coordinates of all sensor nodes on 2-D plane. This is the startup phase. All the GPS equipped sensor nodes exchange their stored dimensions

2. Find Effective Distance Ratio (EDR) calculate at every node. This energy overhead is compensated by extremely large dissemination time interval

$$
\begin{array}{ll}
\mathrm{EDR}= & \begin{array}{l}
\mathrm{n} \\
\mathrm{i}=1
\end{array} \mathrm{dv}(\mid \mathrm{Pc}-\mathrm{Pn}) \mid \\
\mathrm{n} & \sum_{\mathrm{i}=1}^{\mathrm{d}} \mathrm{dv}(|\mathrm{Pa}-\mathrm{Pn}|) \\
\text { Where } \mathrm{dv}=\text { distance vector function } \\
\mathrm{Pi}=\text { Postion of sensor node in consideration } \\
\mathrm{Pn}=\text { Position vector of nth node } \\
\mathrm{Pa}=\text { Position vector of Access Point or Sink } \mathrm{t}
\end{array}
$$

3. The node with the lowest EDR is decided as a cluster head for the first round

4. Broadcast the event from the access point. This should contain the threshold value on which the Sensor Nodes start dissemination to the cluster head. An adhoc dissemination interval can also be fixed to get the real-time data in the network 
5. The cluster node extract the data from backet and store it in memory buffer. When $60 \%$ of the nodes report the threshold then cluster disseminate the data to access point

6. As all the nodes are reporting to the cluster head no dynamic route is required to be established. The LATHAR Agent in this case is developed which takes the data from the network layer to the entire stack of link, mac and NetIf layers and finally to physical layer and the data is propagated with a radio link between the nodes to the cluster head.

\subsection{The Development of LATHAR Protocol}

This routing protocol is developed in ns2 environment and is tested for hierarchical topology. For micaz sensors and varying no of nodes, dissemination time and sensing interval. The sensing, routing and disseminating applications, routing agents, aggregation strategies have been developed in $\mathrm{C}++$ in ns 2 environment. TCL/Tk is used to write the simulation scenario

\section{Simulation Scenario}

The simulation parameter are given as under

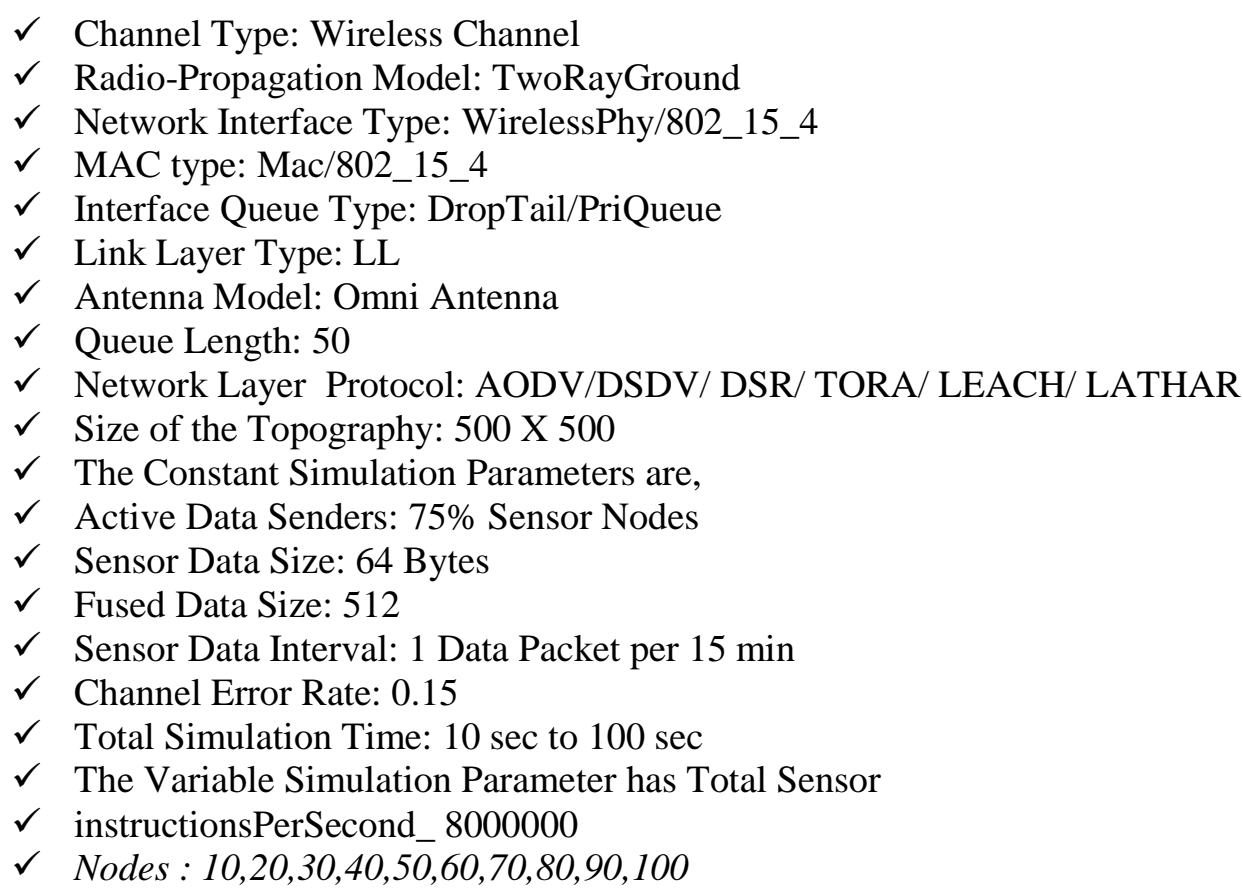

The simulation is performed in ns2 simulator using the TCL script . The trace files obtained from all the protocols in consideration were analysed for various performance parameter. Some of them are given in the result. The behaviour of these parameter on single node as well as the entire network was observed. 


\section{Results}

Simulation Result at the cluster node

Table 1 and Graph1: Packet delivery ratio vs time

\begin{tabular}{|r|r|r|r|r|r|r|}
\hline $\begin{array}{c}\text { Time } \\
\text { in sec }\end{array}$ & AODV & DSDV & DSR & TORA & LEAC & LATHAR \\
\hline 10 & 98.4 & 98.4 & 97.4 & 99.5 & 99.9 & 100 \\
\hline 20 & 100.4 & 97.1 & 98.7 & 98.7 & 99 & 98.7 \\
\hline 30 & 95.2 & 96.1 & 97.2 & 94.3 & 97.1 & 99.3 \\
\hline 40 & 91.9 & 90.3 & 93.1 & 90.5 & 96.7 & 97.9 \\
\hline 50 & 84.5 & 85.9 & 85.5 & 83.3 & 93.2 & 98.4 \\
\hline 80 & 78.2 & 77.5 & 79.6 & 78.9 & 90.4 & 94.9 \\
\hline 70 & 74.2 & 74.2 & 74.8 & 73.1 & 87.9 & 93.3 \\
\hline 80 & 69.9 & 69.6 & 72.5 & 71.6 & 88.3 & 93.4 \\
\hline 90 & 85.9 & 88.8 & 69.6 & 70.2 & 84.7 & 95.1 \\
\hline
\end{tabular}

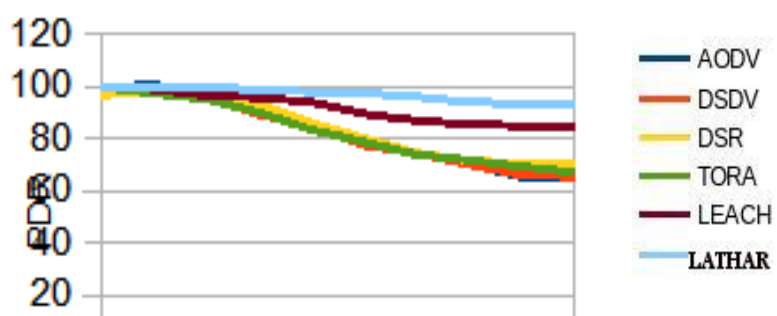

Table 2 and Graph2: Throughput (TIL) vs time

\begin{tabular}{|r|r|r|r|r|r|r|}
\hline $\begin{array}{c}\text { Time } \\
\text { in sec }\end{array}$ & AODV & DSDV & DSR & TORA & LEAC & LATHAR \\
\hline 10 & 400.8 & 482 & 533 & 457 & 541 & 772 \\
\hline 20 & 831 & 825 & 974 & 788 & 1003 & 1181 \\
\hline 30 & 1285.4 & 1102 & 1338 & 1357 & 1608 & 1426 \\
\hline 40 & 1849 & 1673 & 2204 & 1885 & 2213 & 2083 \\
\hline 50 & 2345.2 & 2048 & 2389 & 2378.2 & 2537 & 2280 \\
\hline 60 & 2848.4 & 2844 & 3101 & 2907 & 3044 & 2903.4 \\
\hline 70 & 3035.4 & 2862 & 3029 & 3243 & 3335 & 3175 \\
\hline 80 & 3448.4 & 3377 & 3689 & 3544.4 & 3947 & 3110 \\
\hline 90 & 3978.4 & 3921 & 4077 & 4153 & 4280 & 3147 \\
\hline 100 & 4208.2 & 4087 & 4269 & 4191 & 4432 & 3025 \\
\hline
\end{tabular}

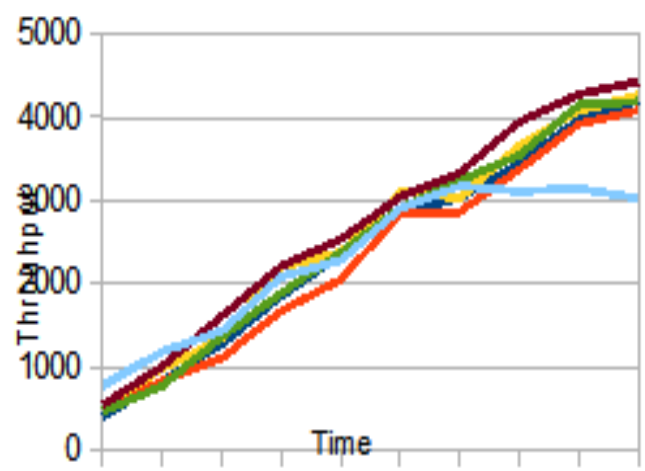

$10203040 \quad 50 \quad 6070 \quad 8090100$

Table 3 and Graph3: Energy Remaining(\%) vs time

\begin{tabular}{|r|r|r|r|r|r|r|}
\hline $\begin{array}{r}\text { Time } \\
\text { in sec }\end{array}$ & AODV & DSDV & DSR & TORA & LE AC & LATHAR \\
\hline 10 & 99.4 & 98.6 & 99 & 100 & 100 & 100 \\
\hline 20 & 99.9 & 98.9 & 98.8 & 97.4 & 95.7 & 100 \\
\hline 30 & 95.1 & 96.1 & 95.1 & 95.8 & 95.8 & 100 \\
\hline 40 & 92.7 & 90.7 & 93.8 & 91 & 97.6 & 99.76 \\
\hline 50 & 85.8 & 84.8 & 88 & 83.2 & 88.8 & 99.01 \\
\hline 80 & 78 & 79 & 82.1 & 79.8 & 89.1 & 98.22 \\
\hline 70 & 75.7 & 72.4 & 76.6 & 74.6 & 88.6 & 98.11 \\
\hline 80 & 70 & 69.8 & 72.1 & 70.8 & 88.8 & 97 \\
\hline 90 & 67 & 65.7 & 71.4 & 65.7 & 84.6 & 97.11 \\
\hline 100 & 87.1 & 65.1 & 68.8 & 68.7 & 85.1 & 97.01 \\
\hline
\end{tabular}

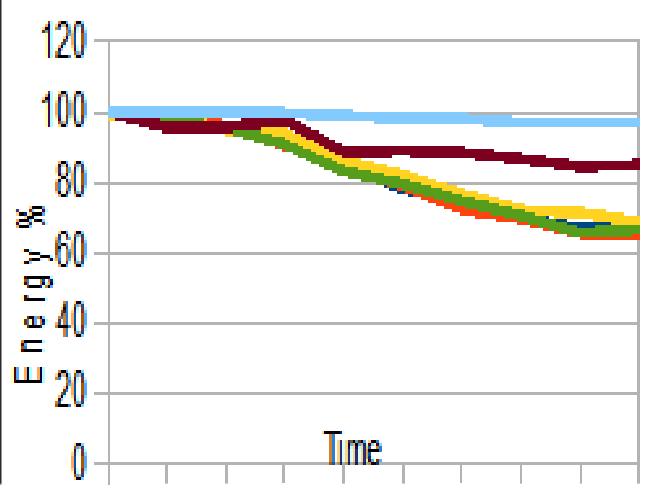

$10 \quad 2030 \quad 40 \quad 50 \quad 6070 \quad 8090100$ 
Simulation Results for different no of nodes in layout

Table 4 and Graph4: PDR vs No of Nodes

\begin{tabular}{|r|r|r|r|r|r|r|}
\hline $\begin{array}{c}\text { No of } \\
\text { Nodes }\end{array}$ & AODV & DSDV & D SR & TORA & LE AC & LATHAR \\
\hline 10 & 58.677 & 58.144 & 57.28 & 59.21 & 59.21 & 59.21 \\
\hline 20 & 59.743 & 58.559 & 58.2 & 57.73 & 58.68 & 59.21 \\
\hline 30 & 56.309 & 57.256 & 57.73 & 58.368 & 57.2 & 59.32842 \\
\hline 40 & 54.532 & 52.934 & 55.38 & 53.407 & 56.72 & 58.38106 \\
\hline 50 & 50.585 & 50.447 & 50.98 & 49.5 & 55.72 & 58.0258 \\
\hline 60 & 46.48 & 45.947 & 47.55 & 46.657 & 53.05 & 57.72975 \\
\hline 70 & 42.213 & 42.156 & 42.16 & 41.817 & 49.16 & 54.30611 \\
\hline 80 & 39.896 & 39.727 & 40.52 & 40.631 & 48.32 & 53.1194 \\
\hline 90 & 36.901 & 37.353 & 39.73 & 39.218 & 48 & 52.83685 \\
\hline 100 & 38.582 & 37.071 & 39.61 & 37.975 & 47.92 & 52.61081 \\
\hline
\end{tabular}

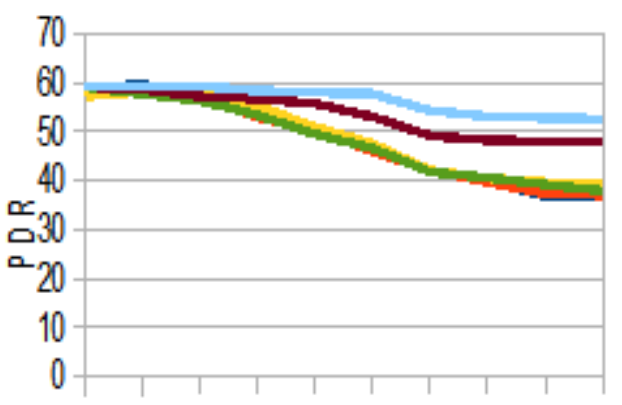

$\begin{array}{llllllllll}10 & 20 & 30 & 40 & 50 & 60 & 70 & 80 & 90 & 100\end{array}$

Table 5 and Graph5: Throughput vs No of Nodes

\begin{tabular}{|r|r|r|r|r|r|r|}
\hline $\begin{array}{r}\text { Time in } \\
\text { sec }\end{array}$ & AODV & DSDV & D SR & TORA & LEAC & LATHAR \\
\hline 10 & 1827.8 & 2510.5 & 3406 & 2013 & 2365 & 5028.678 \\
\hline 20 & 5296.6 & 4936.8 & 4478 & 5143.49 & 6881 & 8143.856 \\
\hline 30 & 8904.7 & 7523.9 & 9101 & 7692.27 & 10409 & 10271.67 \\
\hline 40 & 13540 & 12269 & 16020 & 13693 & 15783 & 15453.43 \\
\hline 50 & 13356 & 14068 & 17828 & 16840 & 18086 & 15698.35 \\
\hline 60 & 17949 & 19403 & 22243 & 21936 & 21324 & 21273.53 \\
\hline 70 & 18310 & 19393 & 20266 & 22532 & 22503 & 21331.97 \\
\hline 80 & 23418 & 23454 & 24896 & 23508 & 27516 & 22107.68 \\
\hline 90 & 24881 & 26967 & 27391 & 29294 & 29645 & 20161.09 \\
\hline 100 & 27896 & 28972 & 29696 & 28716 & 31731 & 20724.58 \\
\hline
\end{tabular}

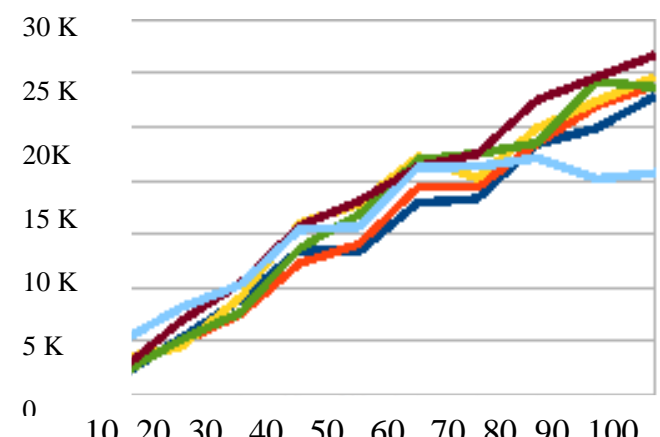

Table 6 and Graph6: Energy Remaining vs No of Nodes

\begin{tabular}{|r|r|r|r|r|r|r|}
\hline $\begin{array}{c}\text { Time } \\
\text { in sec }\end{array}$ & AODV & DSDV & DSR & TORA & LEAC & LATHAR \\
\hline 10 & 60.892 & 60.402 & 60.65 & 61.26 & 61.26 & 61.26 \\
\hline 20 & 61.199 & 60.586 & 60.52 & 59.667 & 58.63 & 61.26 \\
\hline 30 & 58.258 & 58.871 & 58.26 & 58.565 & 58.69 & 61.26 \\
\hline 40 & 56.788 & 55.563 & 57.46 & 55.747 & 59.79 & 61.11298 \\
\hline 50 & 52.439 & 51.826 & 52.68 & 50.968 & 54.4 & 60.65353 \\
\hline 60 & 47.783 & 48.395 & 50.29 & 48.763 & 54.58 & 60.16957 \\
\hline 70 & 44.754 & 42.803 & 45.29 & 44.104 & 52.38 & 58.00263 \\
\hline 80 & 41.384 & 41.266 & 42.63 & 41.739 & 51.32 & 57.3464 \\
\hline 90 & 39.61 & 38.842 & 42.21 & 38.842 & 50 & 57.41143 \\
\hline 100 & 39.67 & 38.487 & 40.67 & 39.433 & 50.31 & 57.35231 \\
\hline
\end{tabular}

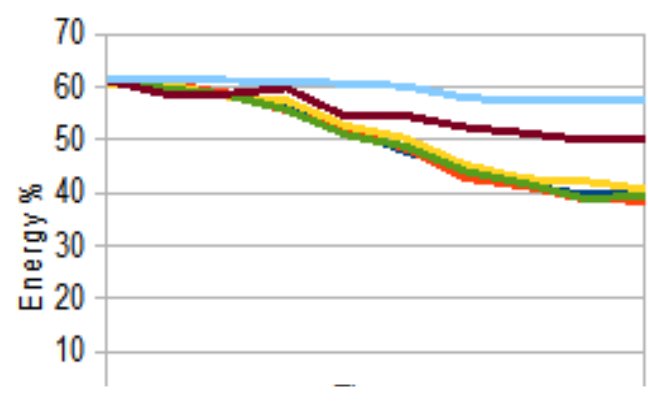

102030405060708090100

\section{Conclusion}

It can be seen that the routing protocol proposed and implemented in simulation gives the best performance. The above results clearly show a higher packet delivery ratio and energy conservation in LATHAR as compared to other protocols, however throughput (comparatively less important measure for our application) decreases at higher time value. This is due to the fact that lower number of packets being transmitted as the event may occur less frequently as compared to other protocols. 


\section{References}

[1] K. Akkaya and A. Younis, "A Survey of Routing Protocols", Ad Hoc Networks 3, (2005), pp. 325-349.

[2] S. Hedetniemi and A. Liestman, "A survey of gossiping and broadcasting in communication networks", Networks, vol. 18, no. 4, (1988), pp. 319-349.

[3] W. Heinzelman, J. Kulik and H. Balakrishnan, "Adaptive protocols for information dissemination in wireless sensor networks", in: Proceedings of the 5th Annual ACM/IEEE International Conference on Mobile Computing and Networking (MobiCom99), (1999); Seattle, WA.

[4] C. Intanagonwiwat, R. Govindan and D. Estrin, "Directed diffusion: a scalable and robust communication paradigm for sensor networks", in: Proceedings of the 6th Annual ACM/IEEE International Conference on Mobile Computing and Networking (MobiCom00), (2000); Boston, MA.

[5] C. E. Perkins and P. Bhagwat, "Highly dynamic destination-sequenced distance-vector routing (DSDV) for mobile computers", ACM SIGCOMM Computer Communication Review, vol. 24, no. 4, (1994).

[6] I. D. Chakeres and E. M. Belding-Royer, "AODV routing protocol implementation design", Distributed Computing Systems Workshops, Proceedings.24th International Conference on IEEE, (2004).

[7] E. M. Royer and C. E. Perkins, "Multicast operation of the ad-hoc on-demand distance vector routing protocol", Proceedings of the 5th annual ACM/IEEE international conference on Mobile computing and networking, ACM, (1999).

[8] D. Johnson, Y. Hu and D. Maltz, "The dynamic source routing protocol (DSR) for mobile ad hoc networks for IPv4", vol. 260, no. 4728, (2007).

[9] W. Heinzelman, A. Chandrakasan and H. Balakrishnan, "Energy-efficient communication protocol for wireless sensor networks", in: Proceeding of the Hawaii International Conference System Sciences, (2000); Hawaii.

[10] A. Manjeshwar and D. P. Agrawal, "TEEN: a protocol for enhanced efficiency in wireless sensor networks", in: Proceedings of the 1st International Workshop on Parallel and Distributed Computing Issues in Wireless Networks and Mobile Computing, (2001); San Francisco, CA.

[11] A. Manjeshwar and D. P. Agrawal, "APTEEN: a hybrid protocol for efficient routing and comprehensive information retrieval in wireless sensor networks", in: Proceedings of the 2nd International Workshop on Parallel and Distributed Computing Issues in Wireless Networks and Mobile computing, (2002); Ft. Lauderdale, FL.

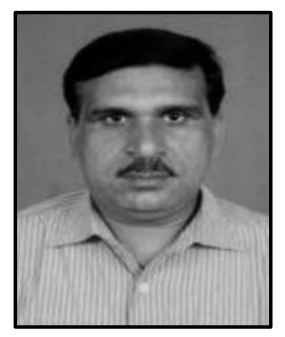

Kamlendu Kumar Pandey has been a post graduate in Computer Applications and Civil Engineering. He 15 plus experience in academics and research. At present he is Asst. Professor in MSc.(IT) VNSGU, Surat and published over 12 research papers in reputed International and National Journal and Conferences. His area of research is wireless sensor Networks and its applications in irrigation. Academically his focus is on Open source software Development for Service oriented and Enterprise applications.

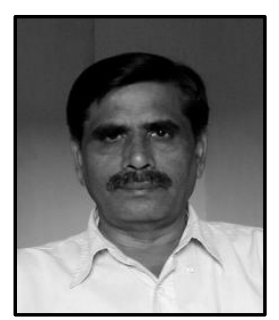

Dr S V Patel (M E, Ph D) is Associate Professor at Computer Science Department, VNSGU, Surat. He has 10 years of experience in industry and 20 years of experience in academics. His areas of interest are Wireless sensor networks, Software Engineering, Computing Architectures. He has more than 20 papers published to his credit. 